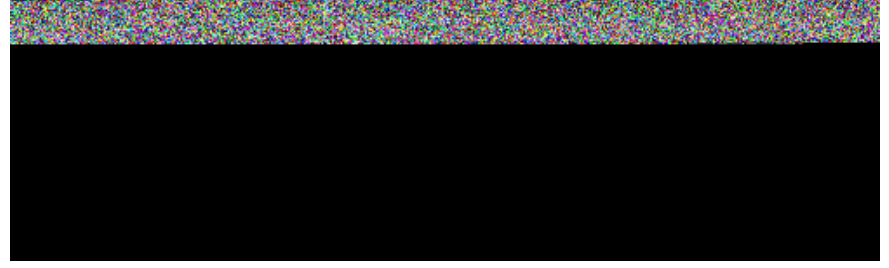

This information is current as of April 26, 2023.

\title{
MR Imaging of Labial Glands
}

M. Sumi, T. Yamada, Y. Takagi and T. Nakamura

AJNR Am J Neuroradiol 2007, 28 (8) 1552-1556 doi: https://doi.org/10.3174/ajnr.A0585

http://www.ajnr.org/content/28/8/1552 


\section{ORIGINAL RESEARCH \\ MR Imaging of Labial Glands}

\author{
M. Sumi \\ T. Yamada \\ Y. Takagi \\ T. Nakamura
}

BACKGROUND AND PURPOSE: The labial salivary gland is a site of occurrence of tumors and cysts, and it serves as the biopsy site for the diagnosis of Sjögren syndrome. However, its imaging features have not been well understood. Here we attempted to depict the labial gland by high-resolution MR imaging.

MATERIALS AND METHODS: The labial glands from 89 patients without Sjögren syndrome, 14 patients with Sjögren syndrome, and 3 patients with tumor or cyst of the lips were imaged by using a 1.5T MR imager with a 47 - or $110-\mathrm{mm}$ surface coil.

RESULTS: The upper and lower labial glands consisted of 1-3 layers of gland clusters, each of which had high signal intensity on T1-weighted and fat-suppressed T2-weighted images and was readily enhanced after gadolinium injection. The posterior parts of the glands were thicker than the anterior parts. The gland areas in the lower lips (186 $\pm 64 \mathrm{~mm}^{2}$ in women and $192 \pm 68 \mathrm{~mm}^{2}$ in men) were greater than those in the upper lips (140 $\pm 46 \mathrm{~mm}^{2}$ in women and $162 \pm 66 \mathrm{~mm}^{2}$ in men). We did not find any significant age-related changes or sex differences in the gland area. The labial gland areas were smaller in the patients with Sjögren syndrome than in patients without Sjögren syndrome, though the difference was significant only in the upper lips $\left(104 \pm 53 \mathrm{~mm}^{2}\right)$.

CONCLUSION: This is the first report describing imaging features of the labial salivary glands. Highresolution MR imaging can readily delineate the labial glands.
$\mathbf{T}$ he minor salivary glands are distributed throughout the anterior two thirds of the tongue, palatal mucosa, buccal mucosa, and lips. The total number of minor salivary glands is estimated to fall into the range of $600-1000 .{ }^{1}$ Collectively, the minor salivary glands produce $6 \%-10 \%$ of the whole saliva. ${ }^{2}$ Saliva, particularly the mucins and secretary immunoglobulin A in minor salivary gland secretions, is considered to play an important role in the protection of the oral mucosa and teeth. ${ }^{1}$ Although the saliva secretion from the minor salivary glands is small in quantity compared with the whole saliva secretion, the broader distributions of the minor salivary glands are advantageous for the protection of the oral cavity against pathogens.

Recently, MR imaging features of the major salivary glands in patients with Sjögren syndrome have been extensively described on the basis of studies using conventional T1- and T2-weighted imaging ${ }^{3,4}$ and diffusion-weighted imaging. ${ }^{5}$ The labial salivary glands have been widely used for the diagnosis of Sjögren syndrome. ${ }^{6}$ In addition, the minor salivary glands, including the labial glands, are the sites for the occurrence of benign and malignant tumors and cysts. However, to our knowledge, the imaging features of healthy or diseased minor salivary glands have not been described.

The paucity of publications of the imaging features of the minor salivary glands may be mainly due to their small size as well as underestimation of the role of the minor salivary glands in maintenance of the oral environment. In the present study, we attempted to delineate the MR imaging features of the healthy labial salivary glands by using small-sized surface coils.

Received September 12, 2006; accepted after revision January 20, 2007.

From the Department of Radiology and Cancer Biology, Nagasaki University School of Dentistry, Nagasaki, Japan.

Please address correspondence to Takashi Nakamura, DDS, PhD, Department of Radiology and Cancer Biology, Nagasaki University School of Dentistry, 1-7-1 Sakamoto, Nagasaki 852-8588, Japan; e-mail: taku@nagasaki-u.ac.jp

DOI 10.3174/ajnr.A0585

\section{Materials and Methods}

\section{Patients}

The study was performed prospectively on 103 patients with or without Sjögren syndrome. The study population included 89 patients without Sjögren syndrome (48 women; average age, 48 years; age range, 11-80 years; and 41 men; average age, 38 years; age range, 7-77 years), who presented for the diagnosis of head and neck disorders but did not have any history or diseases related to the labial glands. We also studied 14 patients with Sjögren syndrome (14 women; average age, 56 years; age range, 29-68 years). The diagnosis of Sjögren syndrome was made on the basis of the European-American criteria. ${ }^{6} \mathrm{We}$ also performed MR imaging on 2 patients with benign tumor (pleomorphic adenomas) and 1 patient with a mucocele. Informed consent for the MR imaging studies of the labial glands was obtained from the 89 patients without Sjögren syndrome, 14 patients with Sjögren syndrome, and the 3 patients with labial mass.

\section{MR Imaging}

MR imaging was performed by using a 1.5T MR imager (Gyroscan Intera 1.5T Master; Philips Medical Systems, Best, the Netherlands) with a 47-mm microscopy coil or 110-mm Synergy flex S Coil (Philips Medical Systems). For MR imaging with a microscopy coil or an $S$ Coil, we used the constant level appearance (CLEAR) postprocessing technique. We obtained conventional T1-weighted (TR/TE/number of signal-intensity acquisitions, $550 / 10 \mathrm{msec} / 3$ for the microscopy coil; TR/TE/number of signal-intensity acquisitions, 500/15 msec/4 for the S Coil) and fat-suppressed (spectral presaturation with inversion recovery [SPIR]) T2-weighted (TR/TE/number of signal-intensity acquisitions, 3140/90 msec/4 for the microscopy coil; TR/TE/ number of signal-intensity acquisitions, $4677 / 80 \mathrm{msec} / 4$ for the $\mathrm{S}$ Coil) axial images of the labial glands by using turbo spin-echo sequences. We used the sensitivity encoding (SENSE) technique for MR imaging with the S Coil. For T1- and T2-weighted imaging with a microscopy coil, we used an $80-\mathrm{mm}$ FOV, 2-mm section thickness, 0.2 -mm section gap, and $160 \times 128$ matrix size. For T1- and T2weighted imaging with the S Coil, we used 180 - to $200-\mathrm{mm}$ FOV, 3 -mm section thickness, $0.3-\mathrm{mm}$ section gap, and $256 \times 224$ matrix 
size. All coils and sequences are commercially available (Philips Medical Systems).

Axial MR images were obtained from all 89 patients without Sjögren syndrome, 14 patients with Sjögren syndrome, and 3 patients with labial masses. Sagittal images were obtained from some patients with Sjögren syndrome or labial masses. We obtained normal anatomic data (size and distribution) on the axial images from the 89 patients without Sjögren syndrome.

For contrast-enhanced studies, gadolinium was injected intravenously at a dose of $0.2 \mathrm{~mL} / \mathrm{kg}$ body weight and at an injection rate of $1.5 \mathrm{~mL} / \mathrm{s}$.

\section{MR Image Analysis}

The maximal thickness and area of each of the upper and lower glands in each patient were determined on a single section of an axial fatsuppressed T2-weighted MR image where the maximal area of the gland appeared. The maximal areas of the labial salivary gland were manually traced on fat-suppressed T2-weighted images by using the ImageJ version 1.29 software program (Wayne Rasband: http://rsb. info.nih.gov/ij/) and were expressed as square millimeter areas. Two radiologists read the images independently in a blinded fashion without knowing the patients' information, and the results obtained by 2 radiologists were averaged.

\section{Statistical Analyses}

Differences in thickness or area between the upper and lower labial salivary glands in the 3 patient groups (women, men, and patients with Sjögren syndrome) were evaluated for their significance by the Wilcoxon signed rank test. Differences in thickness or area between the 2 patient groups were assessed by the Mann-Whitney $U$ test. Agerelated changes were evaluated by the Pearson correlation coefficient. The analyses were performed by using StatView 5.0 software (Abacus Concepts, Berkeley, Calif.).

\section{Results}

The labial glands are localized in the connective tissues of the lamina propria, generally from the mucous membrane to the orbicularis oris muscle, but some glands are found in the muscle layer. MR imaging showed that the glands were hyperintense on T1-weighted and fat-suppressed T2-weighted images (Fig 1). The gland parenchyma was enhanced after gadolinium injection (Fig 2), which was suggestive of rich vascularity of the gland, as was shown in the major salivary glands.

In the upper and lower lips, the glands were thicker in the posterior part (the canine to premolar region) than in the anterior part (the incisor region). Each of the upper labial glands consisted of 1 or 2 layers of clusters of discrete multiple gland areas (Fig $1 A,-B$ ). In the lower lips, each of the glands consisted of 1-3 layers of clusters of discrete gland areas. As a result, the maximal thickness was significantly greater in the lower labial glands $(4.1 \pm 1.1 \mathrm{~mm}$ in women and $4.2 \pm 1.1 \mathrm{in}$ men) than in the upper lips $(2.5 \pm 0.8 \mathrm{~mm}$ in women and $2.9 \pm 1.2$ in men $)(P<.001$ in women and men, Wilcoxon signed rank test). There was no significant difference in gland thickness between women and men (upper labial glands, $P=$ .13 ; lower labial glands, $P=.76$ ). In most cases, the labial glands spanned the left and right first premolars in the upper lips and the left and right second premolars in the lower lips (Fig 1).

We further determined the areas of the glands separately in the upper and lower lips. We did not find any significant sex differences (upper labial glands, $P=.16$; lower labial glands, $P=.55$; Mann-Whitney $U$ test) or age-related changes in gland areas (upper labial glands, $P=.91$ for women and $P=$ .31 for men; lower labial glands, $P=.55$ for women and $P=$ .83 for men; Pearson correlation coefficient). We found that the gland areas in the lower lips $\left(186 \pm 64 \mathrm{~mm}^{2}\right.$ in women and $192 \pm 68 \mathrm{~mm}^{2}$ in men) were greater than those in the upper lips $\left(140 \pm 46 \mathrm{~mm}^{2}\right.$ in women and $162 \pm 66 \mathrm{~mm}^{2}$ in men; Wilcoxon signed rank test) (Fig 3), consistent with the findings of the thickness measurements.

Next, we assessed MR imaging features of the labial glands in patients with Sjögren syndrome (Figs 4 and 5). The gland areas were smaller in the patients with Sjögren syndrome, but the difference was significant only in the upper glands (upper labial glands in patients with Sjögren syndrome, $104 \pm 53$ $\mathrm{mm}^{2}, P=.04$ versus upper labial glands in women without Sjögren syndrome; lower labial glands in patients with Sjögren syndrome, $144 \pm 63 \mathrm{~mm}^{2}, P=.12$ versus lower labial glands in women without Sjögren syndrome; Mann-Whitney $U$ test) (Fig 3).

The high-resolution MR imaging readily depicted pleomorphic adenoma (Fig 6) and mucocele (Fig 7), both derived from the labial salivary glands.

\section{Discussion}

Here we have reported the MR imaging features of the labial salivary glands. To the best of our knowledge, this is the first report describing the MR imaging features of these glands. High-resolution MR imaging by using a small-sized surface coil (47-mm microscopy coil or 110-mm Synergy flex S Coil) readily delineated the labial salivary glands in the upper and lower lips. We have also described MR imaging features of the glands in patients with Sjögren syndrome.

In both the upper and lower lips, the posterior part of the glands was thicker than the anterior part. We found significant differences in the areas of the glands between the upper and lower lips. These findings were consistent with the previous findings that the salivary flow rate of the lower lip salivary gland was higher than that of the upper lip. ${ }^{1}$

Scott ${ }^{7}$ found increasing acinar atrophy and ductal dilation with age. A recent study on the labial salivary glands from autopsy subjects showed that the acinar cell volumes decreased with age, consistent with age-related decreases in labial salivary gland secretion. ${ }^{8}$ The decreases in gland volume were found to be associated with increases in fat tissues in the gland. Therefore, these findings suggest that structural and functional changes occur with age in the labial salivary gland. In the present study, however, we did not find any significant agerelated changes or sex differences in the gland areas. The salivary flow rates of the labial glands were found to be unaffected by age up to 50 years, but thereafter, flow rates decreased in both sexes. ${ }^{1}$ Sonesson et $\mathrm{al}^{9}$ demonstrated that the number of secreting labial glands per unit area decreased with age (children and young adults). However, they also showed that there were no age- or sex-related differences in the salivary secretion rate among the same study groups. In the preceding studies, the labial gland enumeration by using a smear test was limited to the anterior parts of the labial glands. ${ }^{10}$ Therefore, some locoregional differences in age-related changes or sex differ- 

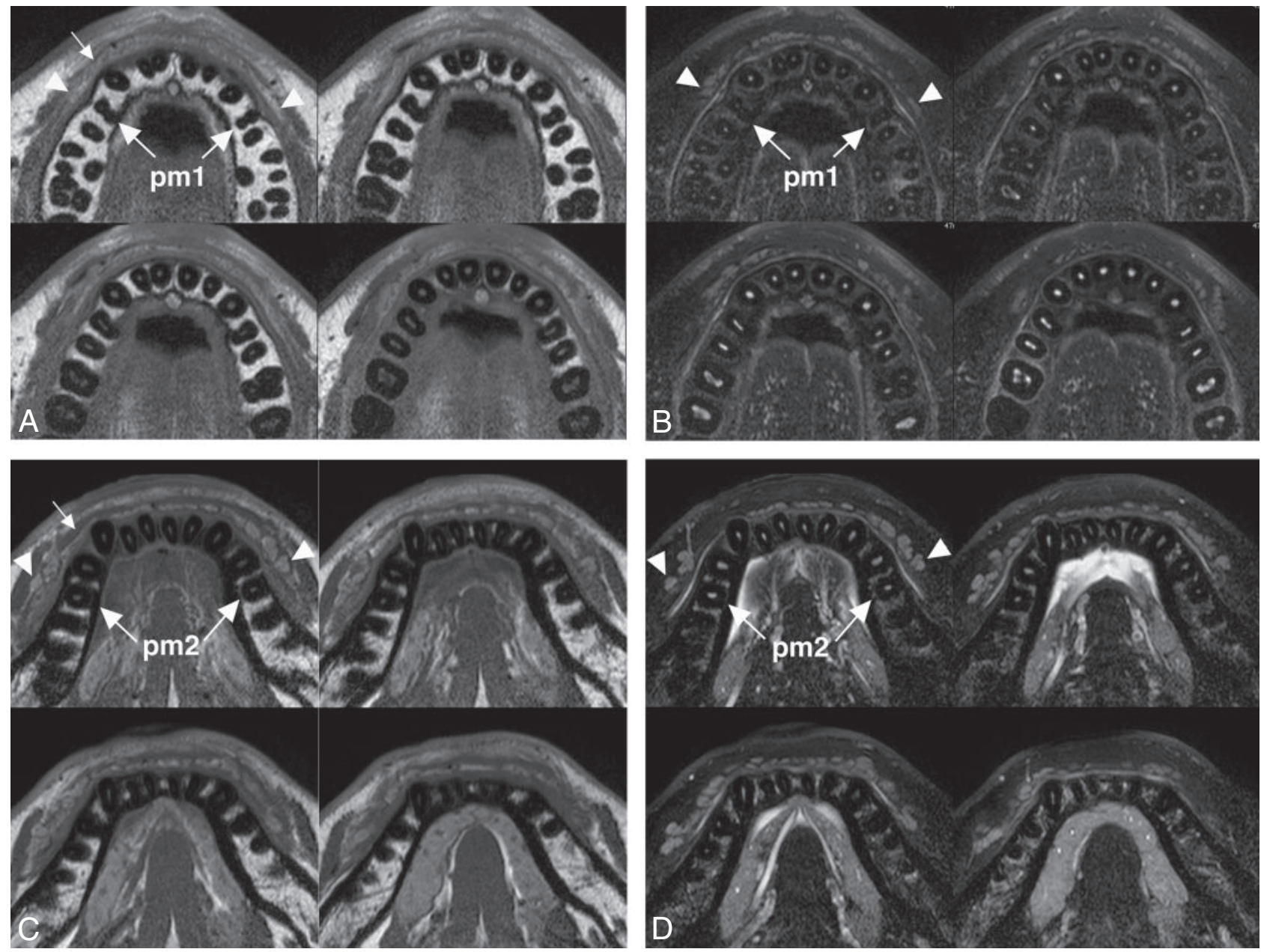

Fig 1. A 26-year-old man with healthy upper and lower labial glands. A, Sequential (upper left to lower right panels in a cephalocaudal direction) axial T1-weighted images using a 47-mm microscopy coil (TR/TE/number of signal-intensity acquisitions, 550/10 msec/3; section thickness, $2 \mathrm{~mm}$; section gap, $0.2 \mathrm{~mm}$ ) of the upper labial salivary gland (arrowheads) show gland clusters spanning the left and right first premolar (pm1) regions. Small arrow indicates orbicularis oris muscle. B, Sequential (upper left to lower right panels in a cephalocaudal direction axial fat-suppressed T2-weighted images using a microscopy coil (TR/TE/number of signal-intensity acquisitions, 3140/90 msec/4) of the upper labial salivary gland (arrowheads) show hyperintense gland clusters consisting of 1 layer in the anterior part and, at most, 2 layers in the posterior part of the lip. pm1 indicates the first premolar. C, Sequential (upper left to lower right panels in a cephalocaudal direction) axial T1-weighted images using a microscopy coil (TR/TE/number of signal-intensity acquisitions, 550/10 msec/3) of the lower labial gland (arrowheads) show gland clusters spanning the left and right second premolars (pm2). Small arrow indicates the orbicularis oris muscle. D, Sequential (upper left to lower right panels in a cephalocaudal direction) axial fat-suppressed T2-weighted images using a microscopy coil (TR/TE/number of signal-intensity acquisitions, 3140/90 msec/4) of the lower labial salivary gland (arrowheads) show hyperintense gland clusters consisting of, at most, 2 layers in the anterior part and of, at most, 2 or 3 layers in the posterior part of the lip. pm2 indicates the second premolar
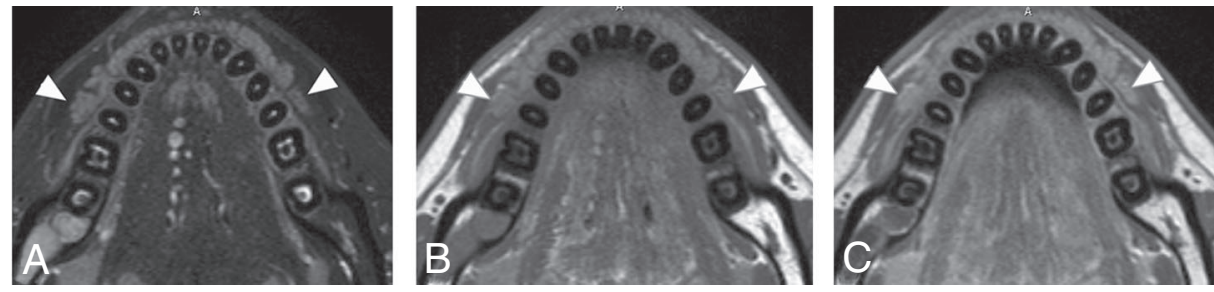

Fig 2. A 22-year-old man with healthy lower labial glands. A, Nonenhanced fat-suppressed T2-weighted image using a 110-mm Synergy Flex S Coil (TR/TE/number of signal-intensity acquisitions, 4677/80 msec/4) shows the discrete contour of lower labial gland parenchyma (arrowheads). B, Nonenhanced axial T1-weighted image using an S Coil (TR/TE/number of signal-intensity acquisitions, 500/15 msec/4) of the lower labial salivary gland shows gland parenchyma (arrowheads) having a signal-intensity slightly higher than that of the muscles C, Gadolinium-enhanced axial T1-weighted image using an S Coil (TR/TE/number of signal-intensity acquisitions, 500/15 msec/4) shows increased signal-intensity levels of the parenchyma of the lower glands (arrowheads), compared with those in $B$.

ences may exist in the labial glands. Further studies using functional imaging of the glands could explain these discrepancies.

Sjögren syndrome is a chronic autoimmune disease characterized by specific lesions in the exocrine glands. ${ }^{10}$ Salivary and lacrimal glands are most frequently and prominently affected by the disease, leading to xerostomia (dry mouth) and xerophthalmia (dry eye), respectively. Biopsies from these glands provide key findings in diagnostic procedures for the disease. ${ }^{10,11}$ The histologic and immunologic changes in the labial salivary glands have been well characterized. Sialographic changes are also characteristic of the disease. However, recent advances in MR imaging techniques could replace 


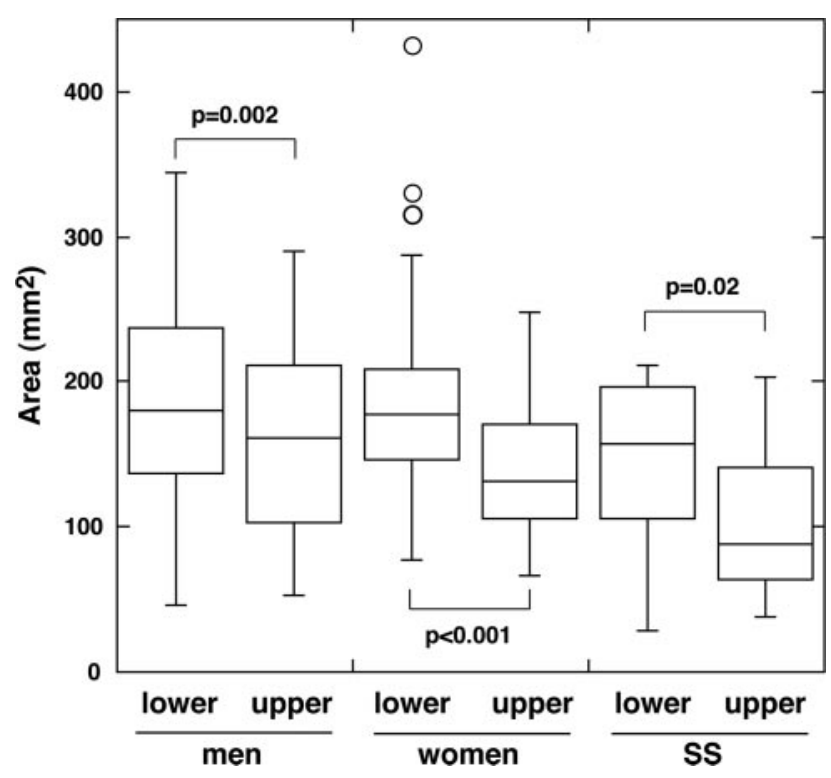

Fig 3. Box plot graph shows areas of healthy upper and lower labial salivary glands in men and women and of upper and lower labial salivary glands in patients with Sjögren syndrome (SS). The horizontal line in each box is a median (50th percentile) of the measured values; the top and bottom of the boxes represent the 25th and 75th percentiles, respectively; and whiskers indicate the range from the largest to smallest observed data points within a 1.5-interquartile range presented by the box $(P$ value, Wilcoxon signed rank test).
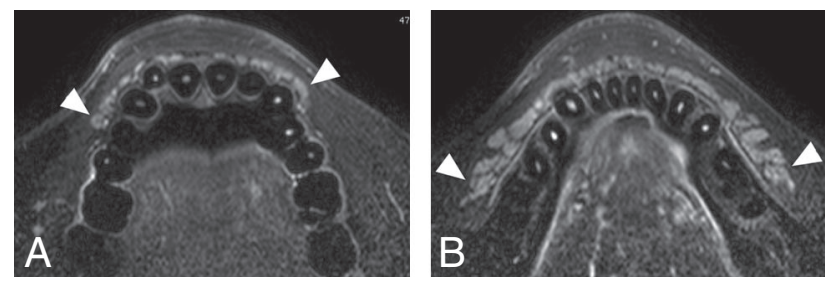

Fig 4. A 55-year-old woman with mild Sjögren syndrome. $A$ and $B$, Axial fat-suppressed T2-weighted images using a microscopy coil (TR/TE/number of signal-intensity acquisitions, $3140 / 90 \mathrm{msec} / 4$ ) of the upper $(A)$ and lower $(B)$ labial salivary glands (arrowheads) show gland areas at a level similar to those in healthy glands.
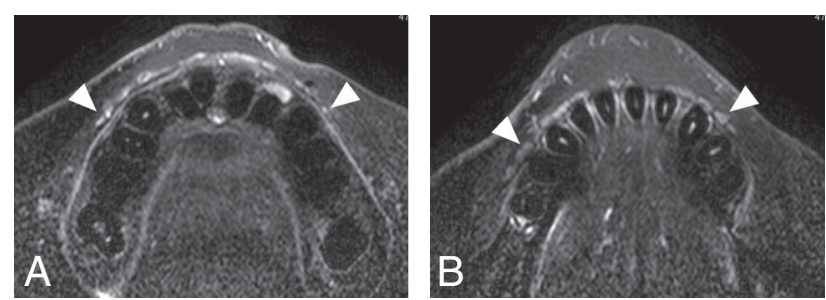

Fig 5. A 53-year-old woman with severe Sjögren syndrome. $A$ and $B$, Axial fat-suppressed T2-weighted images using a microscopy coil (TR/TE/number of signal-intensity acquisitions, $3140 / 90 \mathrm{msec} / 4$ ) of the upper $(A)$ and lower $(B)$ labial salivary glands (arrowheads) show greatly decreased gland areas compared with those in healthy glands.

the classic x-ray sialography in diagnosing Sjögren syndrome. ${ }^{12,13}$ Parenchymal changes were found to be very characteristic. In the present study, we found that the gland areas were smaller in patients with Sjögren syndrome than in patients without the disease. However, the difference was not significant in the lower lips. A comparison between agematched female groups did not reveal any significant differences in gland areas (data not shown).

Previous studies showed that the apparent diffusion coefficients (ADCs) were decreased in the parotid and lacrimal
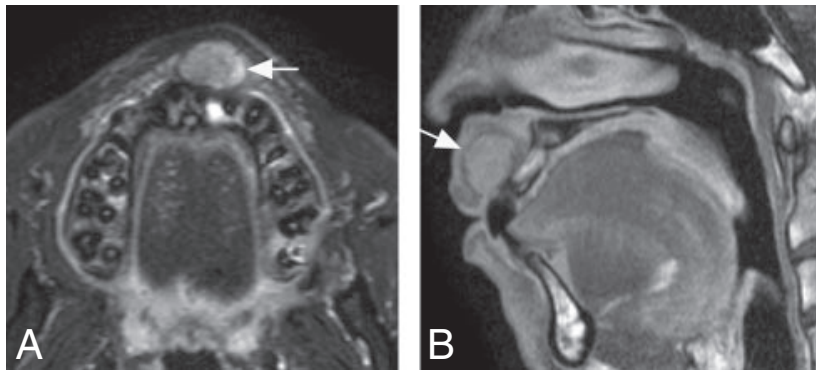

Fig 6. A 29-year-old man with pleomorphic adenoma of upper labial gland. A, Axial fat-suppressed T2-weighted image using an $S$ Coil (TR/TE/number of signal-intensity acquisitions, 4677/80 msec/4) shows a heterogeneous mass (arrow) displacing the anterior portion of the upper labial gland. B, Sagittal fat-suppressed contrast-enhanced T1-weighted MR image using an S Coil (TR/TE/number of signal-intensity acquisitions, 500/15 msec/4) shows a heterogeneously enhanced tumor (arrow).

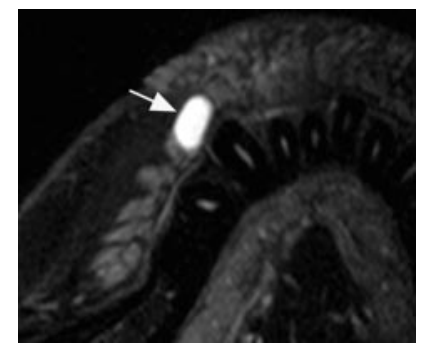

Fig 7. A 31-year-old woman with mucocele in the upper labial gland. Axial fat-suppressed T2-weighted image using a microscopy coil (TR/TE/number of signal-intensity acquisitions, $3140 / 90 \mathrm{msec} / 4$ ) shows a cystic lesion (arrow) with hyperintense signals, deriving from the upper labial gland. The lesion was also imaged by using an S Coil, but this small lesion is better visualized by using the microscopy coil.

glands in patients with Sjögren syndrome. ${ }^{5,14}$ Therefore, we determined the ADCs in the labial glands in patients with or without Sjögren syndrome, expecting lower ADC levels in the labial glands in patients with Sjögren syndrome. However, the small gland sizes and artifacts from dental metals hampered the reproducible measuring of ADCs, and we did not find any significant differences of ADCs between the 2 patient groups.

Among the major disadvantages of using a small-sized surface coil in the imaging of surface organs and tissues of the face are extended image-acquisition times. The image-acquisition time could have been shortened by the use of the parallel imaging technique (SENSE) when a 110-mm Synergy Flex S Coil was used; the image-acquisition time for the upper and lower lips was less than 3 minutes in the present study. In some cases, artifacts from dental metals hampered appropriate assessments of the labial glands, and we excluded such cases from the study population. Image-intensity inhomogeneity may be another shortcoming that is inherent to the use of a smallsized surface coil. To compensate for this, we used the CLEAR postprocessing technique, which enables improved visualization of the deeply located organs and tissues.

An additional limitation of this study may be that we measured the sum of the cross-sectional areas on a single image, instead of the volume of the labial salivary gland. It is likely that some glands were best seen on adjacent sections. However, only 2 or, at most, 3 sections were obtained for each of the lips, and a section that displayed the maximal gland area also contained the maximal length of the gland. Thus, we tentatively used the section displaying the maximum gland area for the determination of the gland size. 
In this study, we performed MR imaging on only 3 patients with tumor or cyst of the labial glands. However, the application of the high-resolution MR imaging to patients with labial tumor or cyst could provide the spatial relationship between the lesions and the glands and thus would be useful for surgeons planning treatment.

\section{Conclusion}

MR imaging by using a small-sized surface coil allowed delineation of the labial glands. We found anatomic differences between the upper and lower labial salivary glands, but age-related changes or sex differences in gland areas were minimal. The upper and lower labial glands consisted of 1 or 2 and 1-3 layers of discrete gland clusters, respectively, and both glands were well vascularized. Future advances in high-resolution MR imaging techniques could allow more definite evaluation of functional and morphologic features of the gland and thus contribute to the diagnosis and treatment planning of salivary gland dysfunctions such as Sjögren syndrome and malignancies of the minor salivary glands in the lips, tongue, palate, and buccal mucosa.

\section{References}

1. Sivarajasingam V, Drummond JR. Measurements of human minor salivary gland secretions from different oral sites. Arch Oral Biol 1995;40:723-29

2. Dawes C, Wood CM. The contribution of oral minor mucous gland secretions to the volume of whole saliva in man. Arch Oral Biol 1973;18:337-42
3. Izumi M, Eguchi K, Ohki M, et al. MR imaging of the parotid gland in Sjogren's syndrome: a proposal for new diagnostic criteria. AJR Am J Roentgenol 1996;166:1483-87

4. Izumi M, Eguchi K, Nakamura $\mathrm{H}$, et al. Premature fat deposition in the salivary glands associated with Sjogren syndrome: MR and CT evidence. AJNR Am J Neuroradiol 1997;18:951-58

5. Sumi M, Takagi Y, Uetani M, et al. Diffusion-weighted echoplanar MR imaging of the salivary glands. AJR Am J Roentgenol 2002;178:959-65

6. Vitali C, Bombardieri S, Moutsopoulos HM, et al. Assessment of the European classification criteria for Sjogren's syndrome in a series of clinically defined cases: results of a prospective multicentre study - The European Study Group on Diagnostic Criteria for Sjogren's Syndrome. Ann Rheum Dis 1996;55:116-21

7. Scott J. Qualitative and quantitative observations on the histology of human labial salivary glands obtained post mortem. J Biol Buccale 1980;8:187-200

8. Vered M, Buchner A, Boldon P, et al. Age-related histomorphometric changes in labial salivary glands with special reference to the acinar component. Exp Gerontol 2000;35:1075-84

9. Sonesson M, Eliasson L, Matsson L. Minor salivary gland secretion in children and adults. Arch Oral Biol 2003;48:535-39

10. Xu KP, Katagiri S, Takeuchi T, et al. Biopsy of labial salivary glands and lacrimal glands in the diagnosis of Sjogren's syndrome. I Rheumatol 1996;23:76-82

11. Lee M, Rutka JA, Slomovic AR, et al. Establishing guidelines for the role of minor salivary gland biopsy in clinical practice for Sjogren's syndrome. J Rheumatol 1998;25:247-53

12. Takagi Y, Sumi M, Sumi T, et al. MR microscopy of the parotid glands in patients with Sjogren's syndrome: quantitative MR diagnostic criteria. AJNR Am J Neuroradiol 2005;26:1207-14

13. Takagi Y, Sumi M, Van Cauteren M, et al. Fast and high-resolution MR sialography using a small surface coil. J Magn Reson Imaging 2005;22:29-37

14. Kawai $Y$, Sumi M, Kitamori H, et al. Diffusion-weighted MR microimaging of the lacrimal glands in patients with Sjögren syndrome. AJR Am J Roentgenol 2005; 184:1320-25 